\title{
Numerical analysis of the interaction between a GTEM-cell and a device under test using a hybrid method
}

\author{
M. Zellerhoff and L. Klinkenbusch \\ Computational Electromagnetics Group, Faculty of Engineering, University of Kiel, Germany
}

\begin{abstract}
Due to the increasing importance of EMC problems through the last years there is a great interest in measurement devices such as GTEM-cells $(\operatorname{Giga}(H z)$ TEM cells). They promise to allow compact and low-cost emission- as well as susceptibility tests up to very high frequencies. Expensive measurement procedures in open-area test sites or within semi-anechoic chambers would become obsolete in many cases. To estimate the quality and reliability of GTEM-cell measurements it is necessary to have detailed knowledge about the processes within the cell and, in particular, about the interactions between the cell and the DUT (device under test). Due to the high frequency and the cell's dimensions a purely numerical simulation while using standard techniques such as Finite Element Method, Method of Moments (MoM) or the Finite-Differ-Time-Domain (FDTD) method is inefficient and unnecessary since the GTEM-cell is a mostly empty homogeneous TEM-waveguide. Analytical models allow only the investigation of empty cells. As will be outlined in the following, a suitable way to reduce the numerical complexity of the general problem is the use of a hybrid method, such as the combination of a modal analysis with the MoM.
\end{abstract}

\section{Two-dimensional model}

To begin with a simple model for the GTEM-cell, we shall analyze the related two-dimensional problem: Consider a perfectly electrically conducting (PEC) DUT, located in the interior of a wedge with PEC walls, where the DUT, the wedge and a (line) source distribution do not depend of the direction parallel to the wedge's edge. This two-dimensional problem is well suited for the evaluation of the algorithm; Moreover it allows an insight into the processes within such a cell, in particular the undesired interaction between the cell and the DUT can be studied systematically. And, it is noted

Correspondence to: Zellerhoff and Klinkenbusch

(zellerhoff@ieee.org; lbk@cem.tf.uni-kiel.de) that the method can be generalized to the three-dimensional case.

\section{Hybrid modal-analysis and method-of-moments ap- proach}

There is only a very small part of the cell occupied by the DUT, while the main part is empty. To take advantage of this fact, we first split the cell into three domains as shown in Fig. 1.

Using a plane polar coordinate system $(R, \varphi)$ with the origin at the wedge's edge, the domains $I, I I$ and $I I I$ are defined as seen from Fig. 1. Domains $I$ and $I I$ are empty, while domain III surrounds the DUT. The electromagnetic field component perpendicular to the plane shown is denoted by $\Psi$ and must satisfy the scalar Helmholtz equation

$$
\Delta \Psi(\boldsymbol{R})+\kappa^{2} \Psi(\boldsymbol{R})=0
$$

within the domains and the Dirichlet- or the Neumanncondition at the boundaries

$$
\begin{array}{r}
\left.\Psi(\boldsymbol{R})\right|_{C}=0 \quad \text { (Dirchlet condition) } \\
\left.\frac{\partial \Psi(\boldsymbol{R})}{\partial n}\right|_{C}=0 \quad \text { (Neumann condition), }
\end{array}
$$

where $\hat{n}$ is the normal outward-directing unit vector at the boundary of the cell. Furthermore, $\Psi$ has to be continuous through the interfaces $C_{1}$ and $C_{2}$ between the domains. A time-factor $e^{+j \omega t}$ is assumed and omitted throughout the analysis.

For Part $I$ and $I I$ of the cell, we describe $\Psi$ by means of a modal analysis

$$
\begin{aligned}
& \Psi^{I}(\boldsymbol{R})=\sum_{n=0}^{\infty} a_{n}^{I i n c} H_{\frac{n \pi}{\alpha}}^{(2)}(\kappa R){ }_{\cos }^{\sin }\left(\frac{n \pi}{\alpha} \varphi\right) \\
& +\sum_{n=0}^{\infty} a_{n}^{I s c} H_{\frac{n \pi}{\alpha}}^{(1)}(\kappa R) \cos _{\cos }^{\sin }\left(\frac{n \pi}{\alpha} \varphi\right)
\end{aligned}
$$




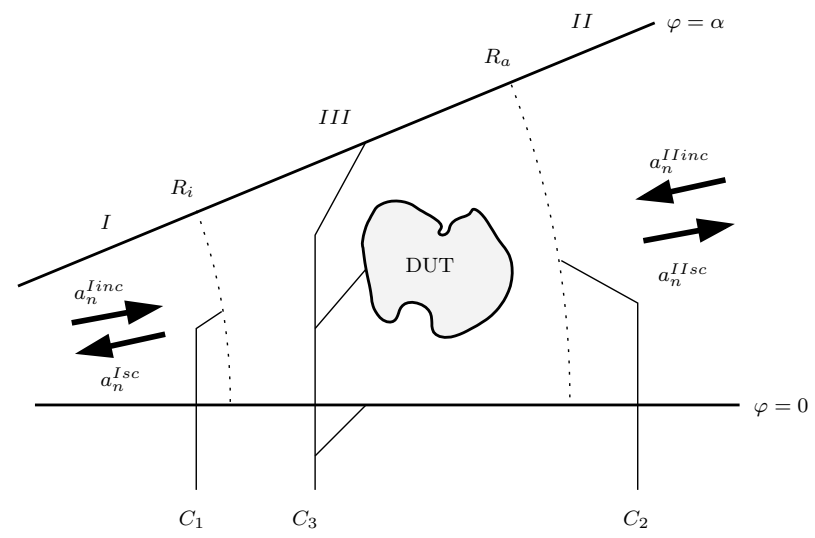

Fig. 1. Subdivision of the cell

$$
\begin{aligned}
& \Psi^{I I}(\boldsymbol{R})=\sum_{n=0}^{\infty} a_{n}^{I I i n c} H_{\frac{n \pi}{\alpha}}^{(1)}(\kappa R) \frac{\sin }{\cos \left(\frac{n \pi}{\alpha} \varphi\right)} \\
& +\sum_{n=0}^{\infty} a_{n}^{I I s c} H_{\frac{n \pi}{\alpha}}^{(2)}(\kappa R) \frac{\sin }{\cos }\left(\frac{n \pi}{\alpha} \varphi\right),
\end{aligned}
$$

where the sine and the cosine functions are to be used for the Dirichlet- and for the Neumann-case, respectively. $H_{\frac{n \pi}{\alpha}}^{(1)}$ and $H_{\frac{n \pi}{\alpha}}^{(2)}$ represent Hankel functions of the first and second kind, respectively, and $\kappa=\omega \sqrt{\varepsilon_{0} \mu_{0}}$ is the free-space wave number. $a_{n}^{\text {I inc }}, a_{n}^{\text {II inc }}$ are the known amplitudes of the incident fields, whereas the amplitudes $a_{n}^{I s c}, a_{n}^{I I s c}$ of the scattered fields are to be determined. While exploiting Green's second identity and provided that there are no sources within domain I I I (susceptibility test) we represent $\Psi$ within domain I I I by

$$
\begin{aligned}
& \oint_{C(F)}\left[G\left(\boldsymbol{R}, \boldsymbol{R}^{\prime}\right) \frac{\partial}{\partial n^{\prime}} \Psi^{\mathrm{III}}\left(\boldsymbol{R}^{\prime}\right)-\Psi^{\mathrm{III}}\left(\boldsymbol{R}^{\prime}\right) \frac{\partial}{\partial n^{\prime}} G\left(\boldsymbol{R}, \boldsymbol{R}^{\prime}\right)\right] d s^{\prime} \\
& =\left\{\begin{array}{ccc}
\Psi^{\mathrm{III}}(\boldsymbol{R}) & \text { for } \quad \boldsymbol{R} \in F \\
\frac{1}{2} \Psi^{\mathrm{III}}(\boldsymbol{R}) & \text { for } & \boldsymbol{R} \in C(F) \\
0 & \text { for } & \boldsymbol{R} \notin F
\end{array}\right.
\end{aligned}
$$

where $C=C_{1}+C_{2}+C_{3}, \hat{n}^{\prime}$ is the normal outwarddirecting unit vector on $C$ and $G\left(\boldsymbol{R}, \boldsymbol{R}^{\prime}\right)$ denotes the freespace Green's function. If the field point in Eq. (5) matches the boundary $C$, we obtain a field integral equation. Since in the Dirichlet case it holds

$$
\left.\oint_{C} G\left(\boldsymbol{R}, \boldsymbol{R}^{\prime}\right) \frac{\partial}{\partial n^{\prime}} \Psi^{\mathrm{III}}\left(\boldsymbol{R}^{\prime}\right) d s^{\prime}\right|_{C_{3}}=0
$$

and in the Neumann case we have

$$
-\left.\oint_{C} \Psi^{\mathrm{III}}\left(\boldsymbol{R}^{\prime}\right) \frac{\partial}{\partial n^{\prime}} G\left(\boldsymbol{R}, \boldsymbol{R}^{\prime}\right) d s^{\prime}\right|_{C_{3}}=\left.\frac{1}{2} \Psi^{\mathrm{III}}(\boldsymbol{R})\right|_{C_{3}}
$$

on the boundary $C_{3}$ only one part of the two in the integrand of Eq. (5) is non-zero in each case; This unknown part is approximated by a set of sub-domain basis functions $f_{i}$ in the following form:

$$
\left.\begin{array}{c}
\left.\Psi^{\mathrm{III}}(\boldsymbol{R})\right|_{C_{3}} \\
\left.\frac{\partial}{\partial n} \Psi^{\mathrm{III}}(\boldsymbol{R})\right|_{C_{3}}
\end{array}\right\}=\sum_{i=1}^{N} c_{i} f_{i} .
$$

On $C_{1}$ and $C_{2}, \Psi^{I I I}$ and $\left(\partial / \partial n^{\prime}\right) \Psi^{I I I}$ are due to the continuity conditions obtained directly as Fourier series from Eq. (3) and (4).

Using the MoM (Harrington, 1993) we transform the problem into a matrix equation. If the coupling-integrals involve only the sub-domain expansions on $C_{3}$, the closed form of the free-space Green's function

$$
G\left(\boldsymbol{R}, \boldsymbol{R}^{\prime}\right)=\frac{1}{4 j} H_{0}^{(2)}\left(\kappa\left|\boldsymbol{R}-\boldsymbol{R}^{\prime}\right|\right) .
$$

is employed, where $H_{0}^{(2)}$ is an Hankelfunction of zero order. The testing on $C_{3}$ is performed with usual point-matching. For the evaluation of the coupling-integrals involving $C_{1}$ and $C_{2}$ we use the modal expansion of the free-space Green's function

$$
\begin{aligned}
& G\left(\boldsymbol{R}, \boldsymbol{R}^{\prime}\right)=\frac{1}{4 j}\left[J_{0}\left(\kappa R_{<}\right) H_{0}^{(2)}\left(\kappa R_{>}\right)\right. \\
& \left.+2 \sum_{n=1}^{\infty} J_{n}\left(\kappa R_{<}\right) H_{n}^{(2)}\left(\kappa R_{>}\right) \cos n\left(\varphi-\varphi^{\prime}\right)\right],
\end{aligned}
$$

with the abbreviations

$$
R_{<}=\min \left(R, R^{\prime}\right) ; \quad R_{>}=\max \left(R, R^{\prime}\right)
$$

and $J_{n}$ as a Bessel function. The testing on $C_{1}$ and $C_{2}$ is performed due to the Galerkin formalism, i.e. testing functions $\cos \frac{n \pi}{\alpha} \varphi$ and $\sin \frac{n \pi}{\alpha} \varphi$ are employed. This procedure allows an analytical evaluation of the related integrals; The convergence is ensured because of the behavior of the cylinder functions and of the coupling-integrals. The numerical solution of the obtained system of linear equations yields the coefficients $c_{i}$ of the sub-domain basis functions on $C_{3}$ and, directly, the scattered-field's modal coefficients $a_{n}^{I s c}, a_{n}^{I I s c}$ in Eq. (3) and (4).

\section{Validation and numerical results}

The consistency of the proposed method has been successfully validated for the case of an empty cell by inserting the problem-adapted Green's function of the wedge, i.e., where the boundary conditions on $C_{3}$ are automatically fulfilled. We analytically derive the expected result: $a_{n}^{I s c}=a_{n}^{I I \text { inc }}$ and $a_{n}^{I I s c}=a_{n}^{I i n c}$. Moreover, this result is also the objective for the numerical evaluation of the empty-cell case: Thus this case is well suited for numerical studies to find the optimal parameters and to estimate the number of relevant modes and sub-domain basis functions.

Figure 2 shows the field inside a cell as compared with the field in free space for a frequency of $5 \mathrm{GHz}$. In Fig. 2 


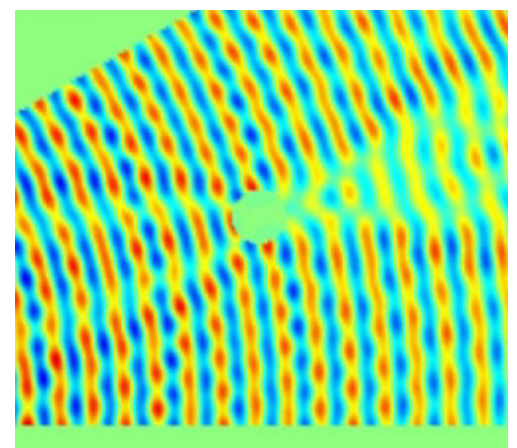

(a)
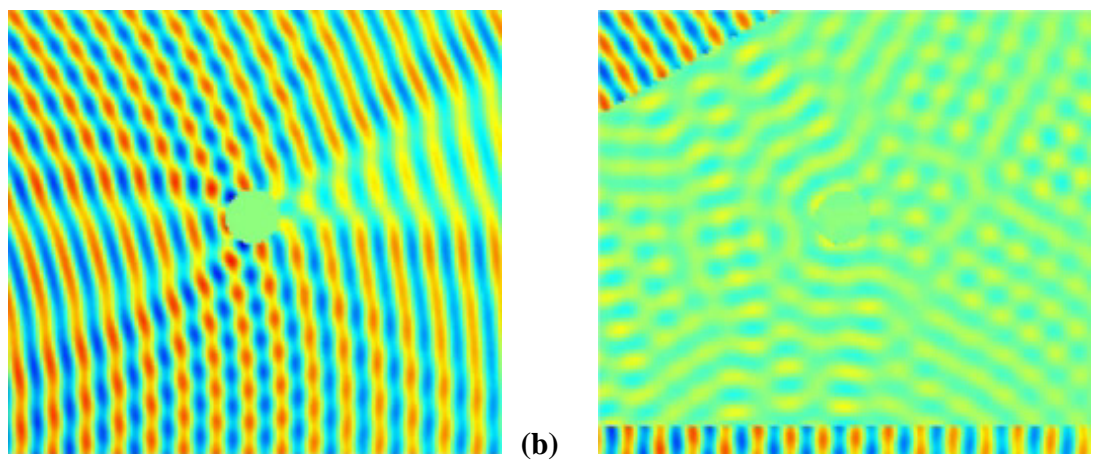

(c)

Fig. 2. Field for an incident TEM-Mode of $5 \mathrm{GHz}$ and for a cylindric scatterer with $5 \mathrm{~cm}$ radius. In (a) the field is calculated inside a cell using the described hybrid method. The length of the cell is $2 \mathrm{~m}$ and the opening angle is $30^{\circ}$. In (b) a reference solution for free space is shown. A multipole expansion has been used for the calculation. The difference between these two results is presented in (c). For both, cell and free space, a Neumann boundary condition is used.

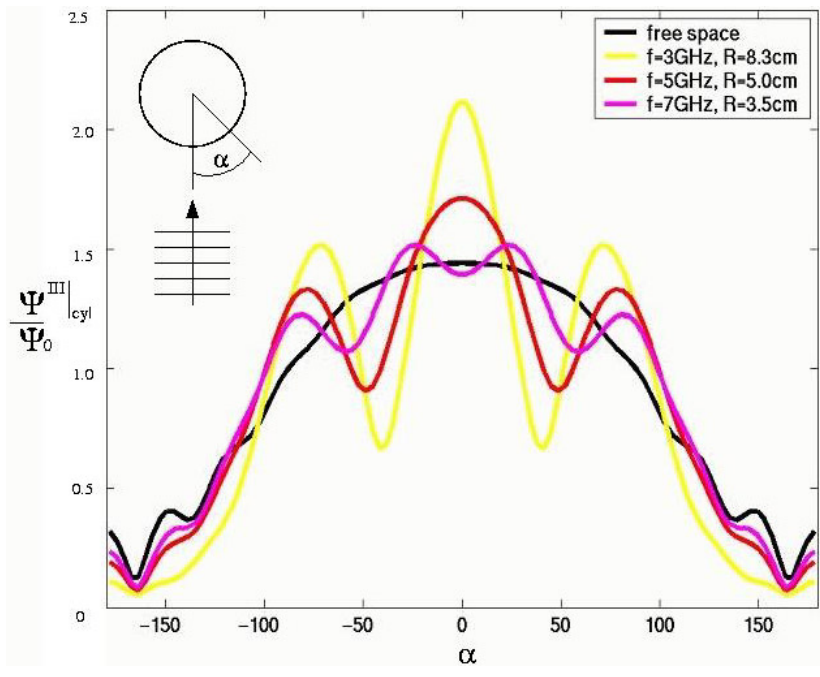

Fig. 3. Normalized surface current on the cylindric scatterer for different frequencies ( $f R=$ const). The source is located in $\alpha=$ $0^{\circ}$ direction. The cylindrical scatterer is located at $R=1.5 \mathrm{~m}$ and $\varphi=15^{\circ}$. The length of the cell is $2 \mathrm{~m}$ and the opening angle is $30^{\circ}$.

we can observe a standing wave between the cell boundaries. This effect causes an error in the surface current as shown in Fig. 3. Figure 4 shows in which way the normalized integral error of the surface current depends on the frequency. This shows that the coupling between the DUT and cell may cause an error in the surface current from $10 \%$ to $60 \%$.

\section{References}

De Leo, R., Pierantoni, L., and Rozzi, T.: Dipole Excitation and Scattering by Spherical Objects in GTEM cells, IEEE Trans. Microwave Theory and Techn., 42, 1700-1708, 1994.

Harrington, R. F.: Field Computation by Moment Methods, IEEE Press and John Wiley \& Sons, New York, 1993.

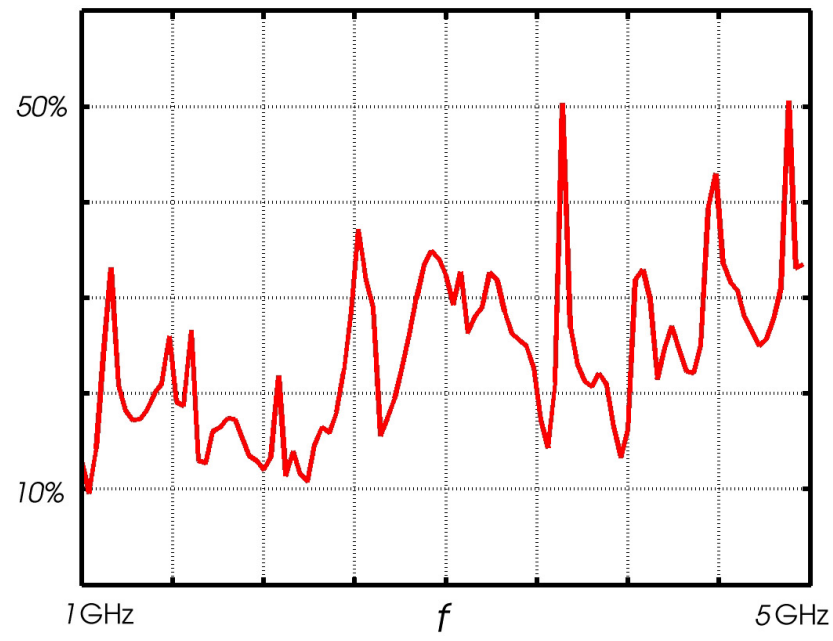

Fig. 4. Normalized quadratic integral error of the surface current $\left(\oint\left|\Psi^{\text {cell }}-\Psi^{\text {free-space }}\right|^{2} d s / \oint\left|\Psi^{\text {free-space }}\right|^{2} d s\right)$ as a function of the frequency. The cylindrical scatterer has a radius of $5 \mathrm{~cm}$ and is located at $R=1.5 \mathrm{~m}$ and $\varphi=15^{\circ}$. The length of the cell is $2 \mathrm{~m}$ and the opening angle is $30^{\circ}$.

Zellerhoff, M., Hannemann, J., and Klinkenbusch, L.: Electromagnetic Coupling between a GTEM-cell and a Device Under Test: A Hybrid Modal-Analysis \& Method-of-Moments Approach, IEEE International Symposium on Antennas \& Propagation and USNC/URSI Meeting, San Antonio, 2002. 